\title{
Dopamine Activates the Motor Pattern for Crawling in the Medicinal Leech
}

\author{
Joshua G. Puhl and Karen A. Mesce \\ Graduate Program in Neuroscience, and Departments of Entomology and Neuroscience, University of Minnesota, Saint Paul, Minnesota 55108
}

\begin{abstract}
Locomotion in segmented animals is thought to be based on the coupling of "unit burst generators," but the biological nature of the unit burst generator has been revealed in only a few animal systems. We determined that dopamine (DA), a universal modulator of motor activity, is sufficient to activate fictive crawling in the medicinal leech, and can exert its actions within the smallest division of the animal's CNS, the segmental ganglion. In the entire isolated nerve cord or in the single ganglion, DA induced slow antiphasic bursting $(\sim 15 \mathrm{~s}$ period) of motoneurons known to participate in the two-step elongation-contraction cycle underlying crawling behavior. During each cycle, the dorsal (DE-3) and ventral (VE-4) longitudinal excitor motoneurons fired $\sim 180^{\circ}$ out of phase from the ventrolateral circular excitor motoneuron $(\mathrm{CV})$, which marks the elongation phase. In many isolated whole nerve cords, DE-3 bursting progressed in an anterior to posterior direction with intersegmental phase delays appropriate for crawling. In the single ganglion, the dorsal (DI-1) and ventral (VI-2) inhibitory longitudinal motoneurons fired out of phase with each DE-3 burst, further confirming that the crawl unit burst generator exists in the single ganglion. All isolated ganglia of the CNS were competent to produce DA-induced robust fictive crawling, which typically lasted uninterrupted for 5-15 min. A quantitative analysis indicated that DA-induced crawling was not significantly different from electrically evoked or spontaneous crawling. We conclude that DA is sufficient to activate the full crawl motor program and that the kernel for crawling resides within each segmental ganglion.
\end{abstract}

Key words: locomotion; Hirudo; central pattern generator; intersegmental coordination; spinal cord; fictive behavior

\section{Introduction}

A major challenge in the field of locomotion is to understand the timing and coordination of body movements that enable an animal to traverse smoothly through its environment. Although sensory feedback is undoubtedly important (Pearson, 2000), the neural basis of locomotion is typically founded on the operation of a central pattern generator (CPG), whereby the CNS generates the timing elements and phase relationships of the movements expressed. Much is known about the neural underpinnings of CPGs in general (Marder and Calabrese, 1996), but far less is understood about pattern generators or the putative "unit burst generators" (Grillner, 1981) that control locomotion in segmented animals. Studies of the rodent spinal cord, lamprey, and additional lower vertebrates (e.g., zebrafish and tadpoles), have contributed significantly to the elucidation of circuitry underlying locomotor control (Grillner, 2006). Invertebrate systems have also been immensely instructive. Examples include swimmeret beating in the crayfish (Mulloney and Hall, 2007), insect flight (Robertson and Pearson, 1985) and swimming in the leech

\footnotetext{
Received Jan. 11, 2008; revised March 22, 2008; accepted March 22, 2008.

This work was supported by National Science Foundation Grant IOB-0523959 (K.A.M.) and fellowships from the National Institutes of Health and 3M (J.G.P.). We thank Kathleen Klukas for the preparation of scientific illustrations and Windy Lynch for assistance with the behavioral studies. We also thank Roger Moon for his advice with the statistical analyses and William B. Kristan Jr for his guidance and enlightening discussions.

Correspondence should be addressed to Dr. Karen A. Mesce, Departments of Entomology and Neuroscience, University of Minnesota, 219 Hodson Hall, 1980 Folwell Avenue, Saint Paul, MN 55108, E-mail: mesce001@umn.edu.

DOI:10.1523/JNEUROSCI.0136-08.2008

Copyright $\odot 2008$ Society for Neuroscience $\quad 0270-6474 / 08 / 284192-09 \$ 15.00 / 0$
}

(Brodfuehrer et al., 1995a). Aside from swimmeret beating (Murchison et al., 1993), the kernel for rhythmic movements is often distributed across segmental units (e.g., flight circuit) (Robertson and Pearson, 1983) or is difficult to elicit and sustain in a single segmental unit. In the leech CNS, for example, serotonin can promote the semblance of the swim motor pattern in a single isolated ganglion, but it is weak and not sustained over time (Hocker et al., 2000). This response contrasts sharply with the robust swimming achieved when a chain of ganglia is stimulated (chemically or electrically) or when intersegmental swim-gating neurons are present (Weeks, 1981).

Ideally, an animal preparation that is competent to produce a reliable, robust, and sustained locomotor rhythm in a single segmental unit would greatly simplify the elucidation of its rhythmogenesis, and serve as a model to understand why some kernels are distributed and some are not. It also could provide a unique system to study intersegmental coordination, as each segmental oscillator would have the capacity for complete independence.

Here, we asked whether the kernel for vermiform crawling was confined to a single segmental division of the CNS. Crawling is a prominent form of locomotion in the leech and consists of the animal releasing its anterior sucker, elongating its body while staying close to the substrate, attaching its anterior sucker, releasing its posterior sucker, and then contracting its entire body with reattachment of the posterior sucker (Gray et al., 1938; Stern-Tomlinson et al., 1986). This cycle is repeated and acts to propel the leech forward. In addition, fictive crawling has been defined in detail (Eisenhart et al., 2000), and key motoneurons have been identified (Baader, 1997). 
In this report, we established how best to activate the crawl CPG in its entirety. We tested whether dopamine (DA), an important neuromodulator of movement, was sufficient to activate crawling. In response to DA application, we describe patterned neural activity obtained from identified motoneurons in preparations that had the entire CNS intact, but isolated from the body. We also examined whether DA could induce fictive crawling in as little as a single isolated ganglion.

\section{Materials and Methods}

Animals. Adult medicinal leeches (species Hirudo medicinalis and $H$. verbana) were obtained from Leeches USA (Westbury, NY) and maintained at room temperature in either Buhl (Buhl, MN) spring water or distilled water containing Hirudo-salt $\left(0.5 \mathrm{~g} \mathrm{~L}^{-1}\right.$; Leeches USA). Leeches used ranged in size between 2.0 and $3.5 \mathrm{~g}$. Leeches were anesthetized on ice or in ice-chilled saline for a minimum of $15 \mathrm{~min}$ before dissection.

Terminology. The leech nervous system consists of a compound cephalic ganglion (i.e., "head brain" that comprises the supraesophageal and subesophageal ganglia), the 21 unfused segmental ganglia (designated M1-M21), and a compound terminal ganglion (see Fig. $1 \mathrm{~A}$, diagram). In this study, the term "whole nerve cord" refers to a complete CNS preparation dissected from its body.

Each segmental ganglion has a dorsal and ventral layer of neuronal somata, and uniquely identified cells often have a functional as well as numerical designator (see Fig. $2 B, C$ ). For example, cell 3 and cell 4 are known, respectively, as the dorsal and ventral longitudinal excitor motoneurons (DE-3 and VE-4, respectively). Cells 1 and 2 are also referred to as the dorsal and ventral longitudinal inhibitor motoneurons (DI-1 and VI-2, respectively). DE-3, which was used as a crawl-burst monitor cell, extends its axon through the dorsal posterior (DP) nerve root.

CNS preparations. After exposing the intact nerve cord, the surrounding sinus was cut dorsally along its entire length and removed from the connectives and ganglia before intracellular recording. The glial sheath was left intact on all preparations recorded. Typically, four DP nerves were left attached to the middle body ganglia (M7-M15) and excess tissue around the nerve root was removed. The CNS was placed into chilled normal saline during dissection and before application of dopamine. Normal saline contained (in $\mathrm{mm}$ ) $116.0 \mathrm{NaCl}, 4.0 \mathrm{KCl}, 1.8 \mathrm{CaCl}_{2}$, $1.5 \mathrm{MgCl}_{2}, 10.0$ dextrose, 10.0 Trizma preset crystals, pH 7.4 (SigmaAldrich, St. Louis, MO) (adapted from Nicholls and Baylor, 1968). Single ganglia were removed from adjacent anterior and posterior ganglia by transecting all connectives; one or both of the DP nerves were left attached.

Electrophysiological recordings and cell identification. To monitor fictive locomotor behaviors, we recorded extracellular unit activity from a given DP nerve using a suction electrode. The signal was amplified using a Grass P15 AC preamplifier (Grass Technologies, West Warwick, RI) and recordings were digitized using a MacLab/4s data acquisition system (ADInstruments, Bella Vista, New South Wales, Australia) attached to a Macintosh personal computer (Apple, Cupertino, CA). Fictive crawling was identified by monitoring the activity of DE-3, which was the largest spike in the DP recording (Kristan et al., 1974). Fictive crawling was defined as three or more consecutive bursts of DE-3 with a period ranging between 5 and $25 \mathrm{~s}$ (Eisenhart et al., 2000). For the calculation of intersegmental burst delays, two extracellular DP-recording electrodes were interposed by one to five ganglia. In some preparations, for example, DP nerves from M9 and M14 or M13 and M16 were recorded, whereas in other preparations, more anterior ganglia (M7 and M9) were recorded. These various recording sites ensured that the intersegmental delays calculated represent a reliable measure of delay constancy.

The intracellular activity of identified neurons was recorded using a Dagan (Minneapolis, MN) IX2-700 amplifier and was digitized as described above. Intracellular electrodes had resistances of $40-65 \mathrm{M} \Omega$ and were filled with a solution of $2 \mathrm{M}$ potassium acetate and $20 \mathrm{~mm}$ potassium chloride. Intracellular electrode tips were typically back filled with $5 \%$ $(\mathrm{w} / \mathrm{v})$ Neurobiotin (Vector Laboratories, Burlingame, CA) dissolved in a $2 \mathrm{~m}$ potassium acetate solution for cell labeling.

All neurons were identified by the stereotypical position and size of their somata, and their electrophysiological signature. Cell identities were further confirmed by iontophoretically injecting $5 \%$ Neurobiotin $(+0.5-1 \mathrm{nA})$ into a given cell for at least $10 \mathrm{~min}$. Tissue was subsequently fixed, processed, treated with $\mathrm{Cy} 3$-conjugated streptavidin (1:50 dilution in PBS for $18-24 \mathrm{~h}$ at $4^{\circ} \mathrm{C}$; Jackson ImmunoResearch, West Grove, PA), and prepared for viewing according to the methods of Gilchrist et al. (1995).

Dopamine superfusion. Dopamine-containing saline was typically delivered into the recording chamber by gravity with a flow rate of $1-2 \mathrm{ml}$ $\min ^{-1}$. Outflow was maintained by passive siphon to preserve fluid levels at 2-3 ml. For some experiments, electric peristalsis pumps were used to introduce and remove the DA solutions. A $1 \mathrm{~mm}$ DA-containing saline solution was made fresh daily, stored in the dark at $4^{\circ} \mathrm{C}$ and used within $4 \mathrm{~h}$. Immediately before use, a portion of the DA concentrate was diluted in normal saline. DA-containing saline was passively warmed to room temperature before superfusion.

Determining effective DA concentrations. To determine which concentrations of DA were most effective for inducing fictive crawling, DA of varying concentrations was superfused onto intact CNS and isolated ganglion preparations. We used DA concentrations of 10, 50, 75, 100, 200 , and $500 \mu \mathrm{M}$. The delivery rate of DA was held at $1-2 \mathrm{ml} \mathrm{min}^{-1}$ for a minimum of $30 \mathrm{~min}$. If we did not observe crawling, as strictly defined above, we considered a given concentration to be ineffective. The degree of effectiveness was determined by the percentage of preparations that exhibited fictive crawling during DA superfusion.

Data analysis and statistical methods. The period of each crawl cycle was determined by calculating the time between midpoints of consecutive DE- 3 bursts. The burst midpoint was calculated by adding the burst onset and 0.5 times the burst duration. The mean crawl period for all DA-induced fictive crawling was calculated by analyzing 10 consecutive crawl cycles. Mean crawl periods for spontaneous or electrically induced fictive crawling were calculated by analyzing $4-10$ crawl cycles. This mean comprised a single sample point in the statistical analyses (i.e., a sample size of $n=10$, representing $100 \mathrm{DE}-3$ bursts). Calculations of crawl periods extracted from published recordings and from spontaneous fictive crawling consisted of three to six periods per sample point. The duty cycle was calculated by dividing the DE-3 burst duration by the coinciding crawl period for that burst.

Parameters of the crawl rhythm described above were compared across three fictive crawl groups. The groups consisted of intact CNS preparations in which crawling was (1) spontaneous or electrically induced or (2) DA induced. The third group consisted of crawling in a single, isolated ganglion induced by DA. We calculated the elongation duration, contraction duration, and period for overt crawling in intact leeches. Crawling leeches were video recorded using a Sony (New York, NY) DCR-TRV840 camcorder. The video was digitized onto a personal computer using Microsoft (Redmond, WA) Moviemaker. The video was analyzed frame by frame to determine the parameters of crawling. Release and reattachment of the head sucker denoted the onset and end of elongation. Release and reattachment of the posterior sucker denoted the onset and the end of contraction. The period of overt crawling was calculated by determining the duration between the onset of elongation and the end of contraction.

All means presented are \pm SEM. Two-tailed, one-way ANOVA tests, Student's $t$ tests, and linear regressions were performed using the software package R (R Development Core Team, 2007) (http://www.Rproject.org.). The confidence level used was $0.95(\alpha=0.05)$. Linear regressions were performed using the least-squared method.

\section{Results \\ Dopamine is sufficient to activate fictive crawling in isolated, intact CNS preparations}

A crawling-like motor rhythm was observed in the DP nerves of 19 of 21 intact CNS preparations that were superfused with 75$100 \mu \mathrm{M}$ DA (Fig. 1C). Other concentrations of DA were tested, but we found $75 \mu \mathrm{M}$ to be optimal for inducing this crawl-like rhythm. In 12 of these 19 preparations (63\%), the crawl-like burst activity propagated through the nerve cord in an anterior-to- 


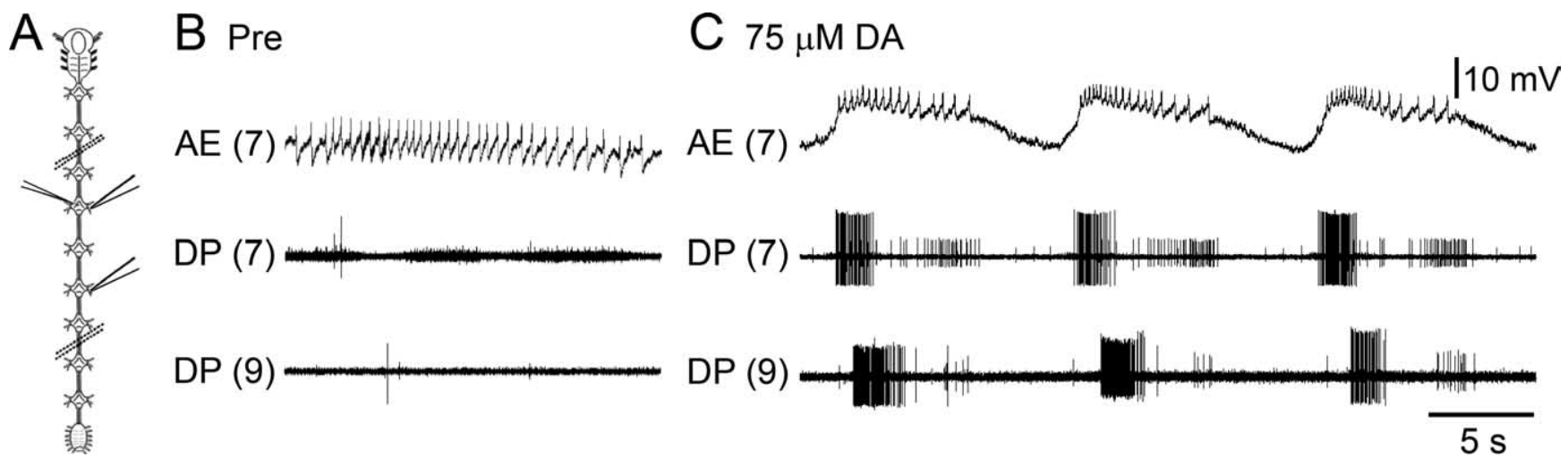

Figure 1. Dopamine-induced fictive crawling in a whole (intact) but isolated CNS. A, Schematic of the leech CNS showing the whole nerve cord and placement of intracellular (small icon) and extracellular (large icon) recording electrodes. Diagonal hatch marks indicate where some of the 21 iterated segmental ganglia are not shown, so as to limit the overall size of the diagram. $\boldsymbol{B}$, Intracellular recording (top) of the AE motoneuron before treatment with DA. Bottom traces are extracellular DP nerve root recordings from ganglia M7 and M9. C, Intracellular recording (top) of the AE motoneuron after superfusion of $75 \mu \mathrm{m}$ DA. Bottom traces are extracellular DP nerve root recordings from ganglia M7 and M9. Calibration in $C$ applies to $\boldsymbol{B}$. Numbers in parentheses denote the segment number.

posterior direction, similar to fictive crawling reported previously (Eisenhart et al., 2000) (Fig. 1C). In the remaining 7 of 19 nerve cords, the DA-induced crawl-like bursts appeared uncoordinated among segments. Of the 12 preparations showing intersegmental burst coordination, five exhibited the anterior-to-posterior propagation during the period of DA application, whereas the other seven showed this propagation only after DA had begun to be washed out of the CNS. Two preparations expressed appropriate propagation during DA application, which eventually subsided, but later resumed during DA washout.

As shown in Figure $1 B$, DP nerve recordings were quiescent before the application of DA, and the annulus erector (AE) motoneuron $(n=4)$ fired tonically. After $\sim 3$ min, crawl-like episodes emerged as indicated by bursting of the largest unit recorded in the DP nerve, the dorsal longitudinal excitor motoneuron (DE-3). Several minutes later, these crawl-like bursts propagated in an anterior-toposterior direction along the nerve cord (Fig. 1C). The intersegmental delay (ISD) between the onset of crawl-like bursts induced during DA superfusion was $0.64 \pm$ 0.09 s per segment $(n=5)$. In nerve cords that exhibited anterior-to-posterior propagation only during DA washout $(n=7)$, and during periods of DA application and

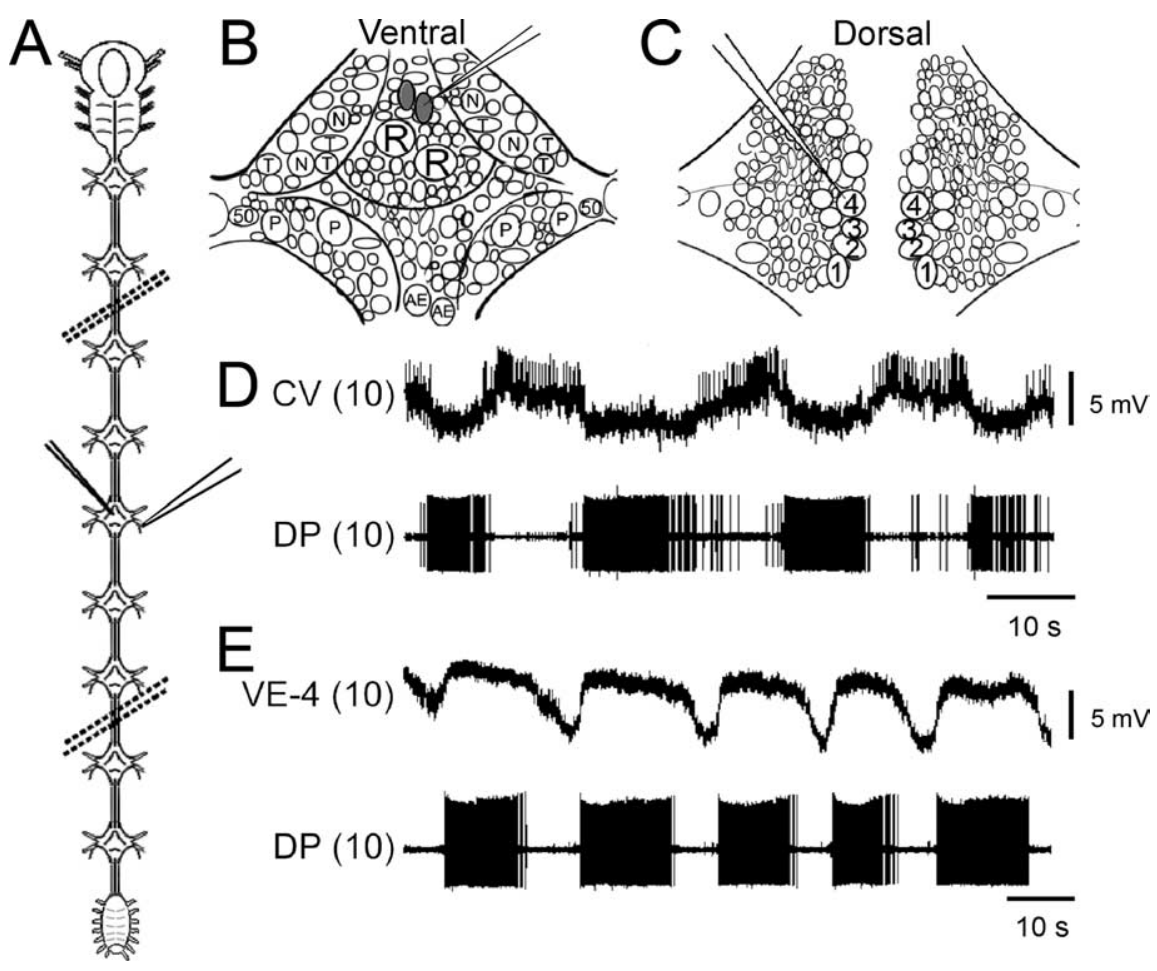

Figure 2. Activities of select motoneurons during dopamine-induced fictive crawling in a whole but isolated CNS.A, Schematic of the CNS of a leech for reference and placement of intracellular (small icon) and extracellular (large icon) recording electrodes. $\boldsymbol{B}$, Schematic of the ventral surface of a typical midbody ganglion, showing placement of the AE motoneurons and the CV motoneurons ( $(V$ filled in with gray). $T, P, N$, and R (Retzius) somata are common landmarks. C, Dorsal surface of a typical midbody ganglion, showing the location of the excitatory and inhibitory motoneurons recorded. D, E, Fictive crawling behavior during $75 \mu \mathrm{M} D A$ superfusion. Top traces are intracellular recordings of the CV (D) and VE-4 (E). Bottom traces are extracellular DP nerve root recordings from the same ganglion in which the intracellular recordings were obtained. The largest unit in the DP nerve recordings belong to motoneuron DE-3. washout $(n=2)$, the ISD was $0.44 \pm 0.08 \mathrm{~s}$ per segment $(n=9)$. Both ISDs were within the range $(0.35-1.15$ s/segment) we calculated using data of spontaneous crawling obtained from studies by Eisenhart et al. (2000) and Briggman and Kristan (2006).

To determine whether the DA-induced pattern of activity we obtained indeed met the established criteria for fictive crawling (Eisenhart et al., 2000), we recorded from motoneurons known to participate in crawling movements (Baader, 1997). A sche-

matic of the experimental preparation, placement of electrodes, and location of somata studied are depicted in Figure $2 A-C$. Using intracellular methods, we recorded from longitudinal and circular motoneurons during DA-induced crawl-like activity. These cells were reported previously to oscillate in phase with either the elongation or contraction steps of fictive crawling (Baader, 1997; Eisenhart et al., 2000). Intracellular recordings indicated that the activity of the ventrolateral circular excitor motoneuron (CV), activated during the elongation step, oscil- 
lated $\sim 180^{\circ}$ out of phase with DE-3 (recorded in the DP nerve), which was active during the contraction step $(n=5)$. CV was hyperpolarized $\sim 2-4 \mathrm{mV}$ and its action potentials were largely suppressed during DE-3 bursting (Fig. 2D). Because the dorsal and ventral longitudinal muscles are coactivated during each contraction step, in contrast to swimming when they are in antiphase, we wanted to establish that the ventral longitudinal excitor motoneurons (e.g., VE-4) fired in phase with DE-3. During DE-3 bursts, VE-4 was depolarized and fired bursts of action potentials that were in phase with DE-3 $(n=4)$ (Fig. $2 E)$. There was a $93.97 \pm 4.95 \%$ overlap between DE-3 bursts and maximal depolarization of VE-4. The out-of-phase activity patterns of the circular and longitudinal motoneurons (CV and DE-3), coupled with the in-phase activity patterns of the longitudinal excitor motoneurons (DE-3 and VE-4) is consistent with previous data that were used to define the crawling motor pattern (Eisenhart et al., 2000).

The fact that DA (in the absence of additional modulators) can activate a fictive locomotor behavior in the dissected CNS is significant, yet it begs the question of where the DA-sensitive pattern generating circuitry resides. Does the pattern-generating kernel reside in a single segmental unit or is it distributed among more than one segmental division? To investigate this question, we conducted experiments using the smallest division of the CNS, the segmental ganglion.

Dopamine activates the crawling motor pattern in an isolated, single ganglion

We observed fictive crawling-like behavior in the isolated, single ganglion after administration of DA (Figs. 3, 4). This rhythm was observed in 95 of 122 (78\%) preparations that were superfused with 75-100 $\mu \mathrm{M}$ DA. Ganglia from the middle body segments $(\mathrm{M} 7-\mathrm{M} 15 ; n=112)$, and from anterior $(\mathrm{M} 2-\mathrm{M} 4 ; n=6)$ and posterior (M16-M21; $n=7$ ) body regions (see below) were all capable of reliably producing fictive crawl-like behavior. The quantitative analysis of this locomotor pattern, however, was primarily conducted on ganglia in the middle body segments M7-M15.

To determine whether the DA-induced pattern of activity in the single ganglion met the established criteria for fictive crawling (aside from intersegmental coordination), we recorded from an ensemble of motoneurons known to define fictive crawling (Baader, 1997; Eisenhart et al., 2000). We analyzed the rhythm of the excitatory longitudinal motoneurons DE-3 and VE-4, the circular motoneuron $\mathrm{CV}$, and the inhibitory longitudinal motoneurons DI-1 and VI-2. In particular, we established that the timing and phase relationships among these motoneurons were indistinguishable from fictive crawling that occurred spontaneously or was electrically induced.

Figure $3 A$ shows an intracellular and extracellular recording of DE-3 during superfusion of $75 \mu \mathrm{M} \mathrm{DA}(n=5)$. DE-3 exhibited a $12-16 \mathrm{mV}$ depolarization with $1-3 \mathrm{mV}$ spikes at the peak of each slow wave. When DE-3 spiking subsided, the membrane potential repolarized to baseline levels that were consistent over time. Individual DE-3 spikes observed in the intracellular recording were correlated with the largest spike in the DP nerve (Fig. $3 A$, shaded areas). This one-to-one relationship reconfirmed that the largest spikes observed in the DP nerve recordings were those of DE-3 and, furthermore, that DE-3 was correctly identified. As presented previously in the intact CNS (see above), VE-4 was active in phase with DE-3 $(n=6)$. In the single ganglion, this cell exhibited a slow depolarization of $10-12 \mathrm{mV}$ with $1-3 \mathrm{mV}$ spikes at the peak of each slow wave (Fig. $3 B$ ). At peak depolarization,
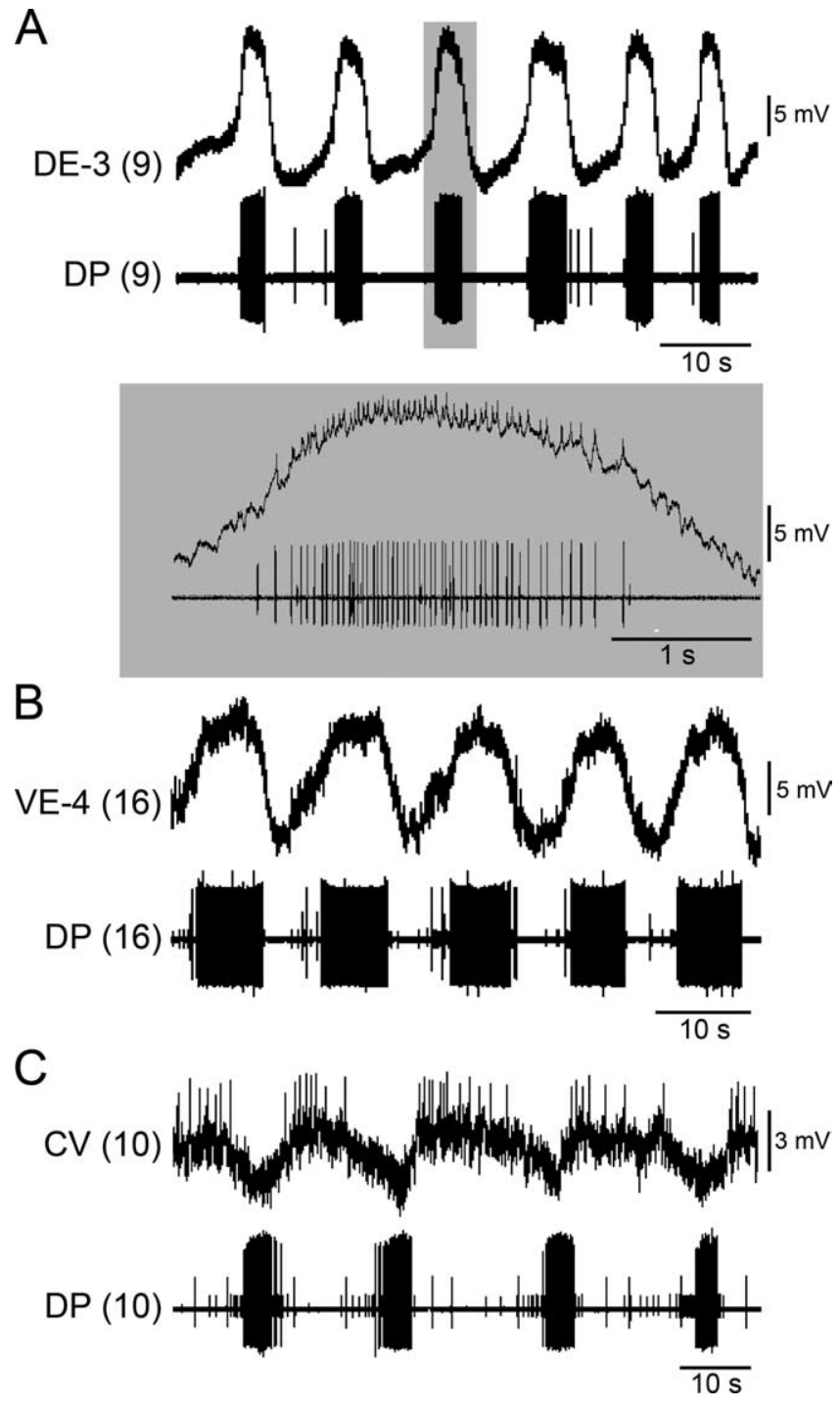

Figure 3. Activities of key excitatory motoneurons during DA-induced fictive crawling in single, isolated ganglia. $\boldsymbol{A}-\boldsymbol{C}$, Top traces are intracellular recordings from motoneurons, and bottom traces are extracellular recordings of the DP nerve, which contains the axon of DE-3, the largest spike in the DP nerve. Numbers in parentheses denote the segment number. $\boldsymbol{A}$, Intracellular and extracellular recording of DE-3, a crawl monitor cell. The two bottom traces (shaded in gray) show an expanded time scale and a 1:1 correlation of spikes. DA concentration was 75 $\mu \mathrm{M} . \boldsymbol{B}, \mathrm{VE}-4 \mathrm{DA}$ concentration was $75 \mu \mathrm{M}$. C, CV DA concentration was $100 \mu \mathrm{m}$.

VE-4 was clearly in phase with each burst of DE-3 (Fig. 3B). In contrast, $\mathrm{CV}$ excitation, which gives rise to elongation, fired rhythmically out of phase with both DE-3 and VE-4 $(n=6)$ (Fig. $3 C$ ). CV peak hyperpolarizations of $2-3 \mathrm{mV}$ were always in phase with the midpoints of the DE-3 bursts. Spikes in CV of $4-6 \mathrm{mV}$ were routinely observed during periods of DE-3 quiescence.

Based on the circular and longitudinal motoneurons being out of phase and the dorsal and ventral excitatory longitudinal motoneurons firing in phase, we suspected that DA was, indeed, activating fictive crawling in the single ganglion. To strengthen our support for such a conclusion, we investigated yet another class of motoneurons, the inhibitory longitudinal motoneurons. These motoneurons are activated to prevent the cocontraction of the CV antagonist muscle groups, i.e., longitudinal muscles (excited by DE-3 and VE-4) (Stuart, 1970). Figure 4, $A$ and $B$, depicts the activities of the inhibitory longitudinal motoneurons DI-1 and VI-2, which fired rhythmically and were phase-locked with step-cycles of the crawl rhythm. Peak 


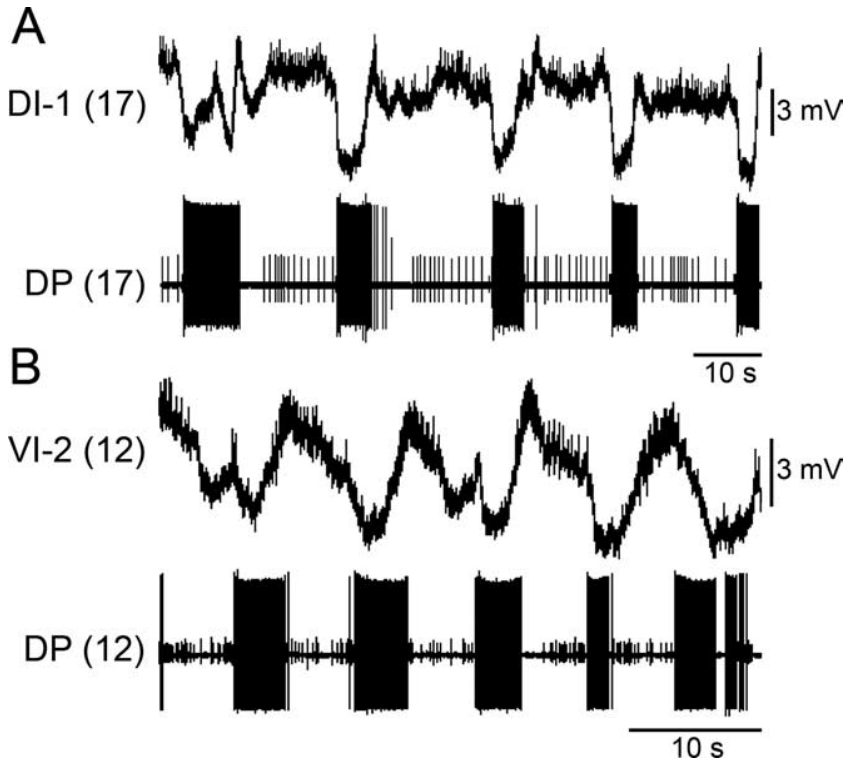

Figure 4. Activities of inhibitory longitudinal motoneurons during DA-induced fictive crawling in single, isolated ganglia. $\boldsymbol{A}, \boldsymbol{B}$, Top traces are intracellular recordings from motoneurons, and bottom traces are extracellular recordings from the DP nerve. Numbers in parentheses denote the segment number. $\boldsymbol{A}, \mathrm{DI}-1 . \boldsymbol{B}, \mathrm{VI}-2$. For $\boldsymbol{A}$ and $\boldsymbol{B}$, the DA concentration was $75 \mu \mathrm{M}$.

excitation of DI-1 correlated with the termination of DE-3 bursts $(n=4)$. This activity is consistent with DI- 1 inhibiting the dorsal longitudinal muscle, i.e., the muscle target of DE-3, thus preventing contraction of the body (Fig. 4A). The motoneuron VI-2, which inhibits the ventral longitudinal muscle target of VE-4, was also rhythmically active and phase-locked with the crawl rhythm $(n=4)$. VI-2 fired action potentials that were out of phase with VE-4 and its maximum hyperpolarization occurred during each DE-3 burst (Fig. $4 B)$.

Last, to document that the far anterior and posterior ganglia were also capable of producing DA-induced crawling, we recorded from DP nerves of single isolated ganglia taken from these body regions. For example, M2 (Fig. 5A) and M19 (Fig. 5B) exhibited DA-induced $(100 \mu \mathrm{M})$ crawling similar to that obtained for the middle body ganglia.

\section{Analysis of specific features of dopamine-induced fictive crawling in the single isolated ganglion}

In addition to analyzing the DA-induced rhythm, we measured the effect of varying DA concentrations, the latency to the onset of fictive crawling and the duration of single bouts of uninterrupted fictive crawling. Figure $6 \mathrm{~A}$ shows the percentage of preparations that produced fictive crawling at $0,10,50,75,100,200$ and $500 \mu \mathrm{M}$ DA. From this, we determined that DA concentrations of $75-100 \mu \mathrm{M}$ were optimal, activating the rhythm in $78 \%$ of preparations $(n=122)$. Slightly lower and higher DA concentrations were able to activate fictive crawling, but were clearly less effective. In particular, preparations exposed to higher DA concentrations showed an initial increase in the tonic firing of DE-3 (monitored in the DP nerve) for $2-5 \mathrm{~min}$, followed by a prolonged quiescence of DE-3 activity and that of all other units in the DP nerve.

Figure $6 B$ shows the latency to onset for DA-induced fictive crawling from 19 randomly selected experiments that were treated with 75-100 $\mu \mathrm{M}$ DA. Whereas the majority of single ganglia exhibited fictive crawling in $<6 \mathrm{~min}$, some preparations required $>9 \mathrm{~min}$. The average latency to onset was $6.0 \pm 2.3 \mathrm{~min}$, which ranged from 0.7 to $18.3 \mathrm{~min}(n=19)$. The majority of DP nerves in DA-treated preparations exhibited a period of quiescence lasting $\sim 30-60$ s before the onset of rhythmic activity. As rhythmic activity emerged, DE-3 bursting, in some preparations, exhibited irregular periods and duty cycles that eventually stabilized, typically within $0.5-1 \mathrm{~min}$.

In addition to the latency to onset of fictive crawling, we calculated the duration of uninterrupted bouts of DA-induced (75$100 \mu \mathrm{M})$ fictive crawling in single isolated ganglia $(n=20)$ (Fig. $6 C)$. The average duration of a single episode was $12.7 \pm 2.6 \mathrm{~min}$, with two crawl episodes lasting $>20 \mathrm{~min}$. Application of higher DA concentrations $(200-500 \mu \mathrm{M})$ resulted in relatively short $(<3$ $\min$ ) latencies to crawl onset, and the duration of uninterrupted crawl bouts was significantly shorter $(3.5 \pm 1.8 \mathrm{~min}, n=3$ of 8 ganglia that crawled; Student's $t$ test, $p<0.012$ ). There were, however, no obvious differences in the period and duty cycle of the ensuing crawl rhythms induced at higher concentrations.

The dopamine-induced fictive crawl rhythm is statistically indistinguishable from spontaneous or electrically induced fictive crawling

To establish whether the DA-induced fictive crawling that we obtained was indistinguishable from fictive crawling that was spontaneous or electrically induced, we measured the period, DE-3 burst duration and duty cycle of the rhythm, and compared these data to published results and data from our experiments. A prominent feature that differentiates the crawl rhythm from other locomotor rhythms, such as swimming, is the period of the DE-3 bursts (Kristan et al., 2005). For the majority of our analyses, we measured the period by calculating the time between burst midpoints (see Materials and Methods). The burst midpoint was used (in contrast to burst onset) to help reduce bias or error when calculating the beginning and ending of DE-3 bursts, although they were usually obvious. Three fictive crawling groups were analyzed and compared: spontaneous or electrically induced fictive crawling (non-DA whole cord), DA-induced fictive crawling in intact CNS preparations (DA whole cord) and DA-induced fictive crawling in single, isolated ganglia (DA single ganglion). Figure $7 A$ shows the mean period for each fictive crawling group and for overt crawling in intact leeches. The average period for the non-DA whole cord group was $19.3 \pm 1.8 \mathrm{~s}$. The average periods for the DA whole cord and the DA single ganglion groups were $14.3 \pm 1.7 \mathrm{~s}$ and $15.5 \pm 1.9 \mathrm{~s}$, respectively. There were no significant differences between the three groups (one-way ANOVA, $p=0.14$ ). The period of overt crawling in intact leeches was $3.3 \pm 0.2 \mathrm{~s}$. This was significantly different (Student's $t$ test, $p<0.001$ ) from the fictive crawling groups and coincides with published results (Eisenhart et al., 2000).

In addition to the period, we compared the duration of DE-3 bursts and the duty cycle across the three experimental groups (described above). The average DE-3 burst durations for the non-DA whole cord, DA whole cord, and DA single ganglion groups were $5.5 \pm 0.9 \mathrm{~s}, 5.1 \pm 0.6 \mathrm{~s}$, and $5.4 \pm 0.6 \mathrm{~s}$, respectively (Fig. $7 B$ ). There were no significant differences between the three groups (one-way ANOVA, $p>0.5$ ). We also calculated the DE-3 duty cycle (ratio of DE-3 burst duration to the coinciding period), because it preserves the relationship of an individual DE-3 burst with its period and not the mean period. The duty cycles for the non-DA whole cord, DA whole cord, and DA single ganglion groups were $0.39 \pm 0.04,0.39 \pm$ 0.03 , and $0.34 \pm 0.02 \mathrm{~s}$, respectively (Fig. 7C). No significant differences existed between these groups (one-way ANOVA, $p=0.45$ ). To compare the duty cycle of fictive crawling to overt crawling, we calculated the ratio of contraction time to the period of overt crawling 

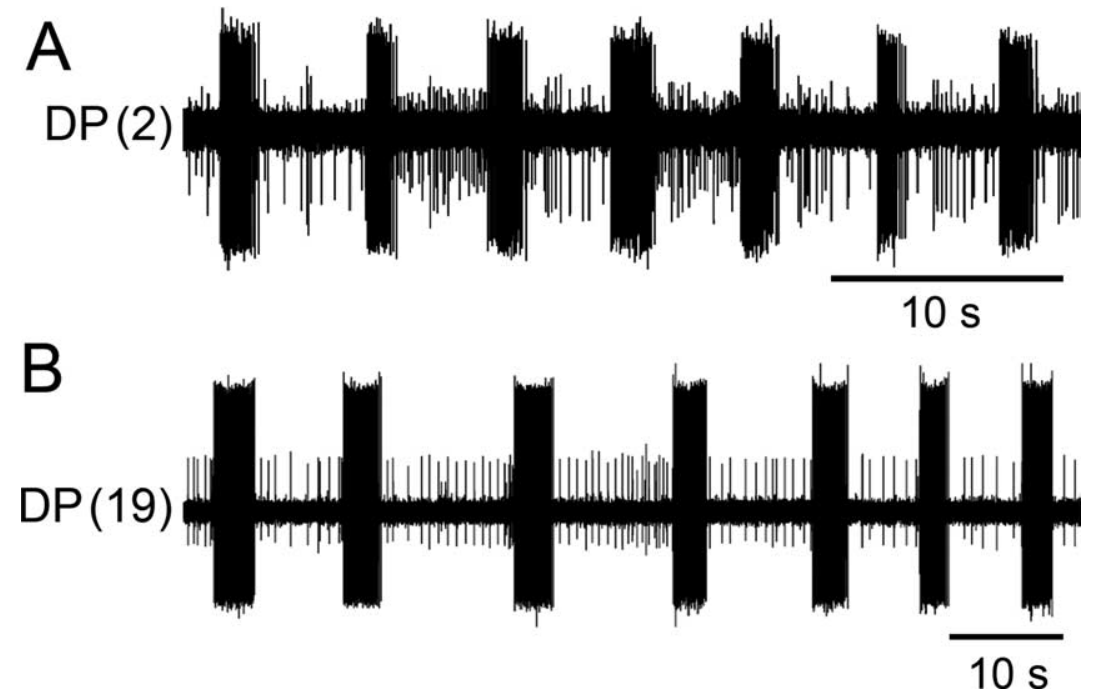

Figure 5. $\boldsymbol{A}, \boldsymbol{B}$, Crawl-like bursting activity of motoneuron DE-3 recorded in the DP nerve of a single isolated ganglion obtained from either an anterior $(\boldsymbol{A})$ or posterior $(\boldsymbol{B})$ body region. Each ganglion was superfused with $100 \mu \mathrm{m}$ DA. $\boldsymbol{A}$, Extracellular recording of the DP nerve root from ganglion M2. $\boldsymbol{B}$, Extracellular recording of a DP nerve root from ganglion M19. Numbers in parentheses denote the segment number.

in intact crawling leeches. We named this ratio the "contraction duty cycle" because DE-3 equates with the contraction step-cycle. The contraction duty cycle for overt crawling was $0.30 \pm 0.01$ (Fig. 7C). No significant differences were found between the duty cycles for the four groups (ANOVA, $p>0.1$ ).

Although the average duty cycles for the three fictive and the overt crawling groups were not significantly different, the question remained whether the duty cycle was maintained throughout the range of crawl periods for the DA-induced fictive crawling groups. To investigate this, we performed a linear regression analysis to determine whether the period of a given DA-induced crawl cycle could predict the duration of the coinciding DE-3 burst. Figure $8 A-C$ shows scatter plots of the mean crawl period versus DE-3 burst duration for each fictive crawl group, with resulting linear regression lines. For comparison, Figure $8 D$ shows the same information for overt crawling. Data from the regression analysis determined that throughout the range of periods for fictive crawling (5-25 s) (Eisenhart et al., 2000), a linear relationship exists between the crawl period and the DE- 3 burst duration (or contraction duration) for DA-induced fictive crawling and for overt crawling. This relationship, however, was strongest for the DA single ganglion and overt crawl groups (Fig. $8 C, D)$.

\section{Discussion}

In this study, we determined that DA activates the motor program for crawling in as little as a single isolated ganglion of the medicinal leech. This is the first report of a singular biogenic amine being sufficient to activate a robust, reliable, and sustained locomotor rhythm in one division of a segmented animal. DA was also found to activate fictive crawling in the isolated whole nerve cord, where appropriate intersegmental coordination between segments could be observed. Based on data obtained in the single ganglion, we can conclude that the rhythm-generating circuitry for crawling exists within the smallest segmental division of the leech CNS, thus empirically defining the unit burst generator for crawling. In addition, because each body segment contains one unique pair of DA-containing neurons, which richly innervates each segmental ganglion or neuromere (Crisp et al., 2002;
Crisp and Mesce, 2004), the release of DA via these neurons probably provides the natural route for the putative actions of DA on the crawl CPG.

Previous studies determined that there was not a significant correlation between the duration of DE- 3 bursts and the period for fictive crawling in isolated CNS preparations (Eisenhart et al., 2000). Our study provides evidence that a linear relationship exists between the duration of DE-3 bursts and the period for fictive crawling induced by DA in the isolated single ganglion (Fig. 8C). A similar correlation also exists for overt crawling, although the periods were shorter (Fig. $8 D$ ). These results suggest that the CPG for crawling possesses intrinsic timing parameters that produce a basic crawling rhythm when unperturbed by outside factors, such as sensory inputs or information from adjacent segments.

\section{Comparisons between crawling and swimming in the leech}

Crawling and swimming are the two most prominent forms of locomotion in the leech, but the mechanisms underlying swimming have received comparatively more attention. Swimming has been thoroughly described at the level of its patterngenerating neural networks (Brodfuehrer and Thorogood, 2001; Brodfuehrer et al., 1995a), gating cells (Kristan and Weeks, 1983), descending command-like cells (Brodfuehrer and Friesen, 1986; Brodfuehrer et al., 1995b; O'Gara and Friesen, 1995; Esch et al., 2002), and modulation by serotonin (Nusbaum and Kristan, 1986; Crisp and Mesce, 2006). Insights into the neuronal bases of both these locomotor patterns can now be advanced by comparing their organization and regulation.

A study by Crisp and Mesce (2004) reported that DA inhibits fictive swimming but not crawling behavior, first suggesting that DA may promote crawling. Because swimming and crawling appear to share rhythm-generating neurons (Esch et al., 2002; Briggman and Kristan, 2006), it may be necessary for DA to suppress components of the swim circuit so that crawling can emerge. Our study indicates that the crawl CPGs, which are the targets of DA modulation, are present in ganglia located in the anterior, middle, and posterior segments of the leech. These results contrast with the segmental nature of the swim CPGs, which appear to be distributed in a more heterogeneous manner. For example, Hocker et al. (2000) reported that short episodes of fictive swimming could be obtained in a fully isolated ganglion taken from middle-body regions, but not so easily from a ganglion residing in anterior body regions. A ganglion from posterior body segments was incompetent to produce any fictive swimming in isolation.

In addition to differences in the competency of anterior versus posterior isolated ganglia to exhibit swim activity, adjacent ganglia and intersegmental neurons are necessary for sustained and robust swimming, in contrast to crawling. Weeks (1981) determined that robust fictive swimming could be induced in nearly isolated ganglia when the two (lateral) connectives were severed, leaving only the small (medial) Faivre's nerve (FN) between adjacent ganglia. The FN contains the axons of the swim gating neurons, cells 204/205, that when depolarized, provide excitatory 
A

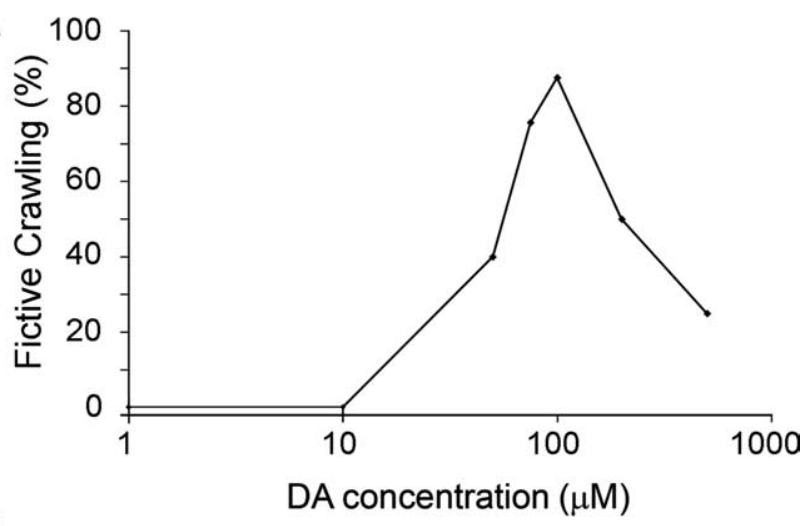

B

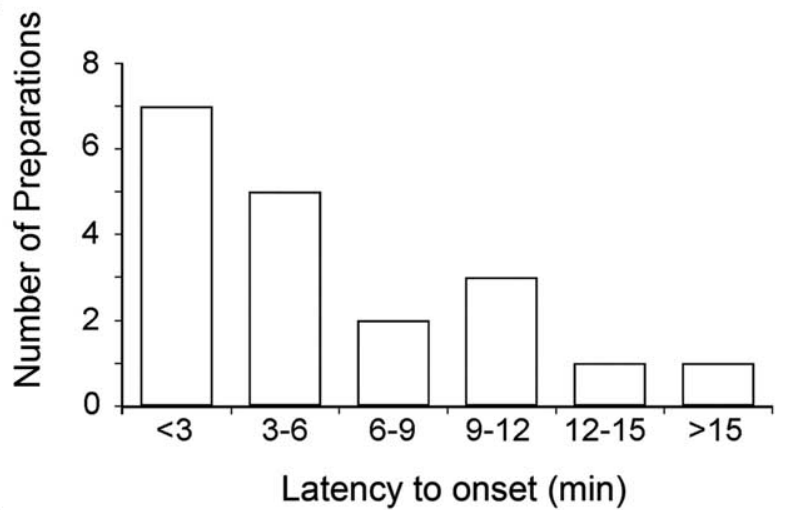

C

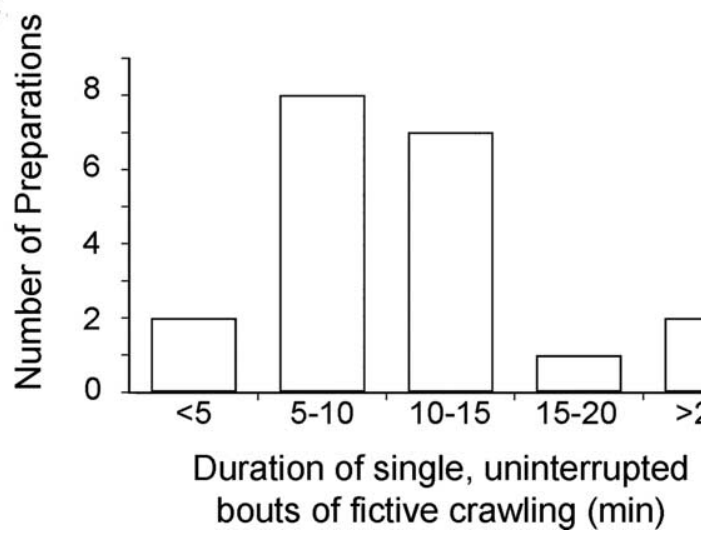

Figure 6. Features of DA-induced fictive crawling in the single, isolated ganglia. $\boldsymbol{A}$, Percentage of single, isolated ganglia that produced fictive crawling in response to varying DA concentrations (log scale). DA concentrations of 75-100 $\mu$ m were determined to be optimal. $B$, Latency to onset of DA-induced fictive crawling in single, isolated ganglia $(n=20)$ superfused with 75-100 $\mu \mathrm{M}$ DA. C, Duration of uninterrupted bouts of DA-induced fictive crawling in single, isolated ganglia $(n=20)$ superfused with $75-100 \mu \mathrm{m} \mathrm{DA}$.

drive to the swim oscillators (Weeks, 1982a,b), activate all other swim-gating neurons, and activate fictive swimming in all FNconnected ganglia for a given length of CNS (Weeks and Kristan, 1978). These data show that although certain ganglia possess a complete oscillator for swimming, these oscillators must rely on excitatory drive from local and adjacent segments for their activation, even in the presence of serotonin. Our study demonstrates that the unit oscillators for crawling are competent to generate crawling in anterior through posterior segmental divisions of the CNS and, after treatment with DA, CPG activation can be sustained without the need for intersegmental drive.

Such organizational differences between swimming and crawling mirror the contrasting nature of these two behaviors. Swimming is a
A

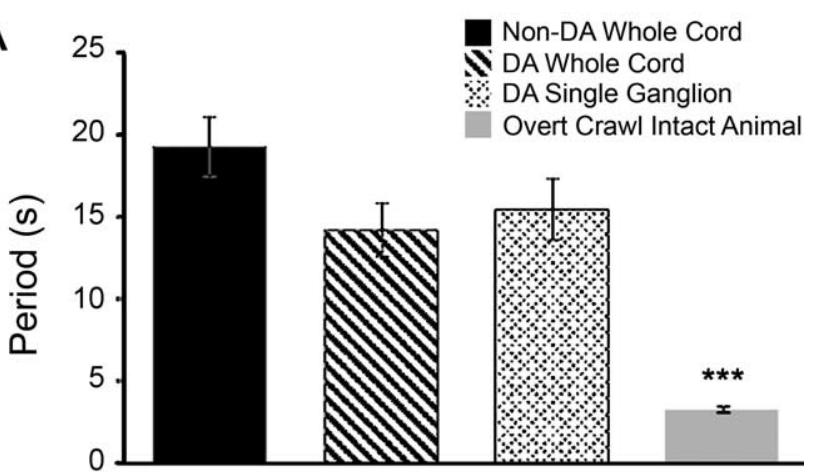

B

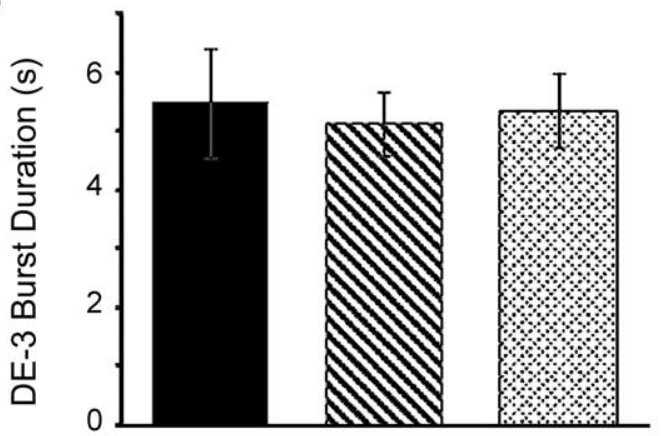

C

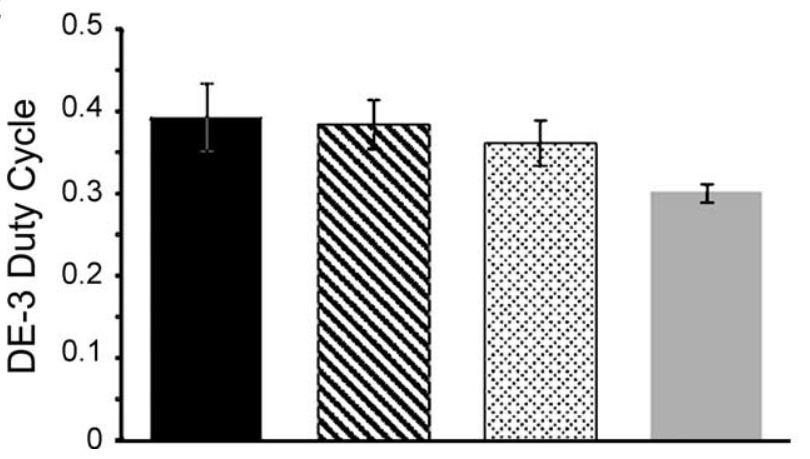

Figure 7. Comparisons of parameters of the crawl rhythm in untreated whole nerve cords (solid black bars), whole nerve cords treated with DA (striped bars), a DA-treated single ganglion (cross-hatch bars), and intact animals (solid gray bars). The sample size for all groups was 10 animals. $\boldsymbol{A}$, Comparison of crawl periods. No statistically significant differences were found between fictive crawl groups (left 3 bars; $p=0.14$ ). A significant difference was found between overt crawling (right bar) and fictive crawling periods ( $\left.{ }^{* * *} p<0.001\right) . B$, Comparison of DE-3 burst durations; no significant differences among the groups were found ( $p>0.5$ ). C, Comparison of DE-3 (contraction) duty cycles. No significant differences among the groups were found $(p>0.1)$.

much faster locomotor behavior $(0.5-2 \mathrm{~Hz})$, and consists of antiphasic dorsal-ventral undulations that propagate along the flattened body with an anterior-to-posterior phase progression, essentially forming a sinusoidal wave that propels the animal forward through its fluid environment. Importantly, the entire length of body must be tightly coordinated throughout each swim cycle to maintain a 3 -phase sinusoidal-like progression of 0,120 , and $240^{\circ}$ (Brodfuehrer et al., 1995a; Zheng et al., 2007). In contrast, during crawling, pauses are commonly observed, especially during the elongation step (Cacciatore et al., 2000; Eisenhart et al., 2000). These pauses are routinely overlaid by other behaviors such as searching movements with the head and whole-body bending (Cacciatore et al., 2000; Mesce et al., 2008) (our unpublished observations). At the conclusion of these subroutines, crawling is often reinitiated during the same step of the crawl cycle before the pause. In order for the leech to suspend and 
A Non-DA Whole Cord

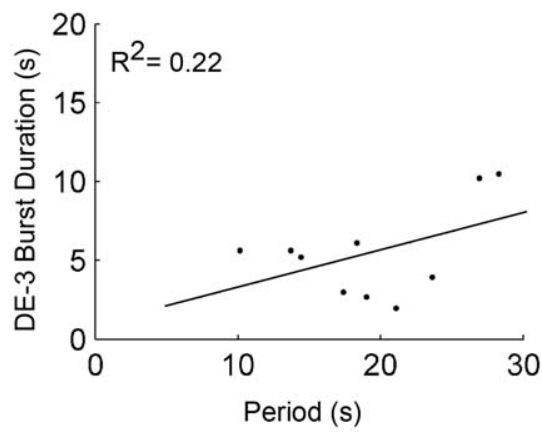

C DA Single Ganglion

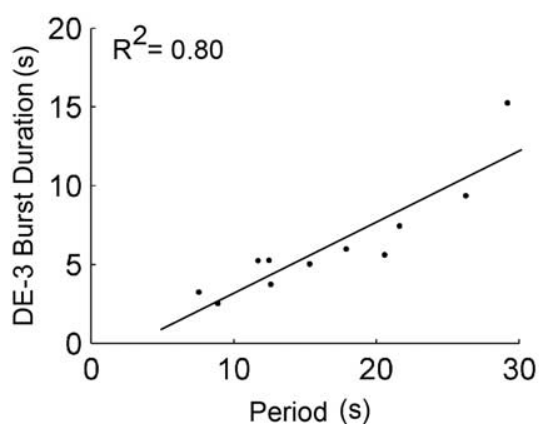

B DA Whole Cord

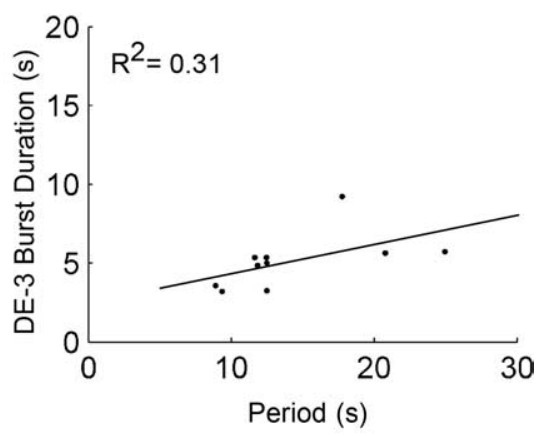

D Overt Crawl Intact Animal

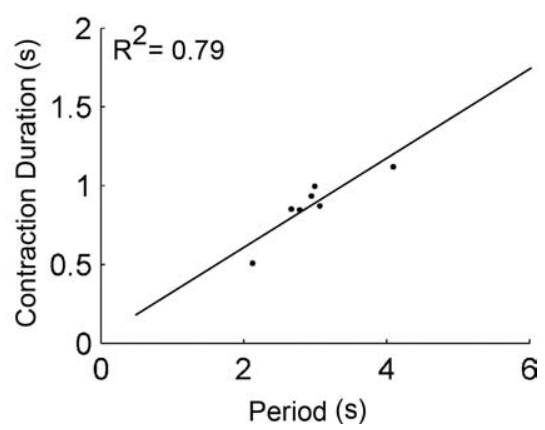

Figure 8. Period dependence of $\mathrm{DE}-3$ burst duration and overt crawl contraction duration. Data points are averaged values from a single animal. Linear regressions were performed using the least squared method $\left(R^{2}\right.$ values are shown in the top left corner of each panel), and the resulting regression lines are shown in each panel. $A-C, D E-3$ burst duration versus the period for the three fictive crawling groups. $\boldsymbol{A}$, Spontaneous or electrically induced fictive crawling in the whole CNS $(n=10)$. The slope of the regression line was 0.24 , and the $y$-intercept was $0.90 . B$, DA-induced fictive crawling in the whole CNS $(n=10)$. The slope of the regression line was 0.19 , and the $y$-intercept was 2.48 . There was a weak relationship between the DE-3 burst duration and the period for fictive crawling in B. C, DA-induced fictive crawling in the single, isolated ganglion $(n=10)$. The slope of the regression line was 0.45 , and the $y$-intercept was -1.30 . D , Contraction duration versus period for overt crawling in intact leeches $(n=10)$. The slope of the regression line was 0.28 , and the $y$-intercept was 0.04 . In $\boldsymbol{C}$ and $\boldsymbol{D}$, a stronger linear relationship existed between the period and the DE-3 burst duration (DA-treated single ganglion) and the contraction duration (intact animal).

reinitiate crawling, the crawl unit oscillators and the coordination among them must be organized differently from those of swimming. This difference likely accommodates segmental independence and flexibility, which probably accounts for our experimental results. The inherent flexibility of crawling behavior, and its two-step (contraction-elongation) locomotor cycle, may thus provide a useful cellular model for understanding adaptive and more complex forms of locomotion in vertebrates (Pearson, 2000; Grillner, 2006).

\section{Comparisons with lower vertebrate locomotion}

Locomotion in many segmented animals share several key features. Typically, locomotor behaviors can be divided into two phases, the power and return strokes (Büschges, 2005). These two phases, generated by the antiphasic activation of opposing, or antagonist, motor pools within the same segment comprise a "unit burst generator" (UBG) (Grillner, 1981). These UBGs are iterated to control each segment of the body or locomotor organ and are coordinated by intersegmental connections (Sigvardt and Miller, 1998; MacKay-Lyons, 2002; Hill et al., 2003). These basic features, which are generated centrally, are found in walking animals such as the stick insect (Büschges, 2005), and in swimming animals such as the Xenopus tadpole and lamprey (Grillner, 2006).

Based on our data, each segmental ganglion of the leech possesses a complete UBG for crawling that includes both excitatory
(Fig. 3) and inhibitory (Fig. 4) motor outputs. The two phases of the UBG produce the elongation (power stroke) and contraction (return stroke) steps of the crawl cycle. How these UBGs are coordinated across segments is not yet understood, but our preliminary data indicate that DAtreated nerve cords will not display appropriate intersegmental coordination if the compound cephalic ganglion is removed (our unpublished observations). Furthermore, the cephalic nervous system appears to be susceptible to declining fluctuations in DA concentrations, explaining why 7 of 12 whole nerve cords expressed coordinated (intersegmental) crawling only during the period of DA washout. These results mirror those of another study, which showed that sustained and robust fictive swimming was activated when serotonin was washed from the compound cephalic ganglion after it had been focally applied (Crisp and Mesce, 2003).

\section{Dopamine is involved in controlling locomotion across animal systems}

The ability of DA to activate locomotion in the leech and other animals supports the idea that DA is part of a conserved and natural route for the activation and regulation of locomotion. In the planarian flat worm, for example, DA is critical for the proper expression of locomotion (Nishimura et al., 2007). In fruit flies that lack the ability to synthesize DA, locomotion is reduced or abolished (Pendleton et al., 2002). In cases where DA release has been induced, for example, in the newt (Matsunaga et al., 2004) and lamprey (Svensson et al., 2003), locomotion is altered. In the larval zebrafish, the blockade of DA receptors (using clozapine) reduces overall locomotion (Boehmler et al., 2007).

In mammals, DA can induce slow locomotor-like rhythms, for example, as in the lumbar regions of isolated neonatal rat spinal cords (Barriere et al., 2004). This property of DA that induces slow and rhythmic locomotor-related bursting parallels our observations in the leech. To achieve a behaviorally relevant locomotor pattern in rodents, however, a mixture of neuroactive substances often needs to be administered (Whelan et al., 2000).

The notion that amines can affect CPGs directly is supported by studies in the lamprey where serotonin acts directly on interneurons of the swimming CPG during glutamate-induced rhythmogenesis (Wallen et al., 1989). Although a number of studies support the idea that DA significantly contributes to locomotor control (Svensson et al., 2003), it has been unclear, to date, if DA activates a locomotor CPG directly. Our current study provides supporting evidence that DA can, indeed, activate the CPG for crawling in the smallest segmental unit possible, i.e., the single ganglion housing the unit burst generator for crawling. Because locomotion in the leech possesses many of the basic features of vertebrate locomotion, it provides a viable and accessible system to understand conserved mechanisms for locomotor pattern generation, intersegmental coordination and modulation by DA. 


\section{References}

Baader AP (1997) Interneuronal and motor patterns during crawling behavior of semi-intact leeches. J Exp Biol 200:1369-1381.

Barriere G, Mellen N, Cazalets JR (2004) Neuromodulation of the locomotor network by dopamine in the isolated spinal cord of newborn rat. Eur J Neurosci 19:1325-1335.

Boehmler W, Carr T, Thisse C, Thisse B, Canfield VA, Levenson R (2007) D4 dopamine receptor genes of zebrafish and effects of the antipsychotic clozapine on larval swimming behaviour. Genes Brain Behav 6:155-166.

Briggman KL, Kristan Jr WB (2006) Imaging dedicated and multifunctional neural circuits generating distinct behaviors. J Neurosci 26:10925-10933.

Brodfuehrer PD, Friesen WO (1986) Initiation of swimming activity by trigger neurons in the leech subesophageal ganglion. I. Output connections of Tr1 and Tr2. J Comp Physiol A Neuroethol Sens Neural Behav Physiol 159:489-502.

Brodfuehrer PD, Thorogood MS (2001) Identified neurons and leech swimming behavior. Prog Neurobiol 63:371-381.

Brodfuehrer PD, Debski EA, O’Gara BA, Friesen WO (1995a) Neuronal control of leech swimming. J Neurobiol 27:403-418.

Brodfuehrer PD, Parker HJ, Burns A, Berg M (1995b) Regulation of the segmental swim-generating system by a pair of identified interneurons in the leech head ganglion. J Neurophysiol 73:983-992.

Büschges A (2005) Sensory control and organization of neural networks mediating coordination of multisegmental organs for locomotion. J Neurophysiol 93:1127-1135.

Cacciatore TW, Rozenshteyn R, Kristan Jr WB (2000) Kinematics and modeling of leech crawling: evidence for an oscillatory behavior produced by propagating waves of excitation. J Neurosci 20:1643-1655.

Crisp KM, Mesce KA (2003) To swim or not to swim: regional effects of serotonin, octopamine and amine mixtures in the medicinal leech. J Comp Physiol A Neuroethol Sens Neural Behav Physiol 189:461-470.

Crisp KM, Mesce KA (2004) A cephalic projection neuron involved in locomotion is dye coupled to the dopaminergic neural network in the medicinal leech. J Exp Biol 207:4535-4542.

Crisp KM, Mesce KA (2006) Beyond the central pattern generator: amine modulation of decision-making neural pathways descending from the brain of the medicinal leech. J Exp Biol 209:1746-1756.

Crisp KM, Klukas KA, Gilchrist LS, Nartey AJ, Mesce KA (2002) Distribution and development of dopamine- and octopamine-synthesizing neurons in the medicinal leech. J Comp Neurol 442:115-129.

Eisenhart FJ, Cacciatore TW, Kristan Jr WB (2000) A central pattern generator underlies crawling in the medicinal leech. J Comp Physiol A Neuroethol Sens Neural Behav Physiol 186:631-643.

Esch T, Mesce KA, Kristan WB (2002) Evidence for sequential decision making in the medicinal leech. J Neurosci 22:11045-11054.

Gilchrist LS, Klukas KA, Jellies J, Rapus J, Eckert M, Mesce KA (1995) Distribution and developmental expression of octopamine-immunoreactive neurons in the central nervous system of the leech. J Comp Neurol 353:451-463.

Gray J, Lissman HW, Pumphrey RJ (1938) The mechanism of locomotion in the leech (Hirudo medicinalis). J Exp Biol 15:408-430.

Grillner S (1981) Control of locomotion in bipeds, tetrapods and fish. In: Handbook of physiology (Brookhart JM, Mountcastle VB, eds), pp 11791236. Bethesda, MD: American Physiological Society.

Grillner S (2006) Biological pattern generation: the cellular and computational logic of networks in motion. Neuron 52:751-766.

Hill AA, Masino MA, Calabrese RL (2003) Intersegmental coordination of rhythmic motor patterns. J Neurophysiol 90:531-538.

Hocker CG, Yu X, Friesen WO (2000) Functionally heterogeneous segmental oscillators generate swimming in the medical leech. J Comp Physiol A Neuroethol Sens Neural Behav Physiol 186:871-883.

Kristan Jr WB, Weeks JC (1983) Neurons controlling the initiation, generation and modulation of leech swimming. Symp Soc Exp Biol 37:243-260.

Kristan Jr WB, Stent GS, Ort CA (1974) Neuronal control of swimming in the medicinal leech I. Dynamics of the swimming rhythm. J Comp Physiol 94:97-119.

Kristan Jr WB, Calabrese RL, Friesen WO (2005) Neuronal control of leech behavior. Prog Neurobiol 76:279-327.

MacKay-Lyons M (2002) Central pattern generation of locomotion: a review of the evidence. Phys Ther 82:69-83.

Marder E, Calabrese RL (1996) Principles of rhythmic motor pattern generation. Physiol Rev 76:687-717.
Matsunaga M, Ukena K, Baulieu EE, Tsutsui K (2004) 7alphahydroxypregnenolone acts as a neuronal activator to stimulate locomotor activity of breeding newts by means of the dopaminergic system. Proc Natl Acad Sci USA 101:17282-17287.

Mesce KA, Esch T, Kristan Jr WB (2008) Cellular substrates of action selection: a cluster of higher-order descending neurons shapes body posture and locomotion. J Comp Physiol A Neuroethol Sens Neural Behav Physiol, in press.

Mulloney B, Hall WM (2007) Local and intersegmental interactions of coordinating neurons and local circuits in the swimmeret system. J Neurophysiol 98:405-413.

Murchison D, Chrachri A, Mulloney B (1993) A separate local patterngenerating circuit controls the movements of each swimmeret in crayfish. J Neurophysiol 70:2620-2631.

Nicholls JG, Baylor DA (1968) Specific modalities and receptive fields of sensory neurons in CNS of the leech. J Neurophysiol 31:740-756.

Nishimura K, Kitamura Y, Inoue T, Umesono Y, Sano S, Yoshimoto K, Inden M, Takata K, Taniguchi T, Shimohama S, Agata K (2007) Reconstruction of dopaminergic neural network and locomotion function in planarian regenerates. Dev Neurobiol 67:1059-1078.

Nusbaum MP, Kristan Jr WB (1986) Swim initiation in the leech by serotonin-containing interneurones, cells 21 and 61. J Exp Biol 122:277-302.

O'Gara BA, Friesen WO (1995) Termination of leech swimming activity by a previously identified swim trigger neuron. J Comp Physiol A Neuroethol Sens Neural Behav Physiol 177:627-636.

Pearson KG (2000) Neural adaptation in the generation of rhythmic behavior. Annu Rev Physiol 62:723-753.

Pendleton RG, Rasheed A, Sardina T, Tully T, Hillman R (2002) Effects of tyrosine hydroxylase mutants on locomotor activity in Drosophila: a study in functional genomics. Behav Genet 32:89-94.

R Development Core Team (2007) R: a language and environment for statistical computing. Retrieved April 3, 2008, from http:// www.r-project.org.

Robertson RM, Pearson KG (1983) Interneurons in the flight system of the locust: distribution, connections, and resetting properties. J Comp Neurol 215:33-50.

Robertson RM, Pearson KG (1985) Neural circuits in the flight system of the locust. J Neurophysiol 53:110-128.

Sigvardt KA, Miller WL (1998) Analysis and modeling of the locomotor central pattern generator as a network of coupled oscillators. Ann NY Acad Sci 860:250-265.

Stern-Tomlinson W, Nusbaum MP, Perez LE, Kristan Jr WB (1986) A kinematic study of crawling behavior in the leech, Hirudo medicinalis. J Comp Physiol A Neuroethol Sens Neural Behav Physiol 158:593-603.

Stuart AE (1970) Physiological and morphological properties of motoneurones in the central nervous system of the leech. J Physiol (Lond) 209:627-646.

Svensson E, Woolley J, Wikstrom M, Grillner S (2003) Endogenous dopaminergic modulation of the lamprey spinal locomotor network. Brain Res 970:1-8.

Wallen P, Christenson J, Brodin L, Hill R, Lansner A, Grillner S (1989) Mechanisms underlying the serotonergic modulation of the spinal circuitry for locomotion in lamprey. Prog Brain Res 80:321-327.

Weeks JC (1981) Neuronal basis of leech swimming: separation of swim initiation, pattern generation, and intersegmental coordination by selective lesions. J Neurophysiol 45:698-723.

Weeks JC (1982a) Synaptic basis of swim initiation in the leech. I. connections of a swim-initiating neuron (cell 204) with motor neurons and pattern-generating "oscillator" neurons. J Comp Physiol A Neuroethol Sens Neural Behav Physiol 148:253-263.

Weeks JC (1982b) Segmental specialization of a leech swim-initiating interneuron, cell 2051. J Neurosci 2:972-985.

Weeks JC, Kristan Jr WB (1978) Initiation, maintenance and modulation of swimming in the medicinal leech by the activity of a single neuron. J Exp Biol 77:71-88.

Whelan P, Bonnot A, O’Donovan MJ (2000) Properties of rhythmic activity generated by the isolated spinal cord of the neonatal mouse. J Neurophysiol 84:2821-2833.

Zheng M, Friesen WO, Iwasaki T (2007) Systems-level modeling of neuronal circuits for leech swimming. J Comput Neurosci 22:21-38. 